\title{
ALMOST CONTACT METRIC AND METALLIC RIEMANNIAN STRUCTURES
}

\author{
Gherici BELDJILALI
}

Laboratory of Quantum Physics and Mathematical Modeling (LPQ3M), University of Mascara, ALGERIA

\begin{abstract}
The metallic structure is a fascinating topic that continually generates new ideas. In this work, new metallic manifolds are constructed starting from both almost contact metric manifolds and we obtain some important notions like the metallic deformation. We give a concrete example to confirm this construction.
\end{abstract}

\section{INTRODUCTION}

Manifolds equipped with certain differential-geometric structures possess rich geometric structures and such manifolds and relations between them have been studied widely in differential geometry. Indeed, almost complex manifolds, almost contact manifolds and almost product manifolds and relations between such manifolds have been studied extensively by many authors.

The differential geometry of the Golden on Riemannian manifolds is a popular subject for mathematicians. In 2007, Hrețcanu [12 introduced the Golden structure on manifolds and in [14] the geometry of the golden structure on manifolds was studied. Now, Such manifolds have been studied by various authors (see [3, 5, 11, 15, 16]). Later, the author in [3] gave a set of techniques to construct many compatible well-known structures on a Riemannian manifold, starting from a Golden Riemannian manifold. And also he established in [4 an interesting class of almost Golden Riemannian manifolds such as the s-Golden manifolds.

As generalization of the Golden mean, the metallic means family appear in 1997 by Vera W. de Spinadel (see [10]) which contains the silver mean, the bronze mean, the copper mean and the nickel mean, etc. The metallic mean family plays an important role in establishing a relationship between mathematics and architecture.

2020 Mathematics Subject Classification. Primary 53C26; Secondary 53D15.

Keywords and phrases. Almost contact metric structure, metallic Riemannian structure.

घherici.beldjilali@univ-mascara.dz

(D) 0000-0002-8933-1548.

(C)2020 Ankara University Communications Faculty of Sciences University of Ankara-Series A1 Mathematics and Statistics 
For example, silver and golden mean can be seen in the sacred art of India, Egypt, China, Turkey and different ancient civilizations. Now, there are also several recent works in this direction [13, 14, 7] and others. Recently, a new type of structure on a differentiable manifold is studied in [9] and the relation between metallic structure and almost quadratic $\varphi$-structure is considered in [17].

Here we show that there exists a correspondence between the metallic Riemannian structures and the almost contact metric structures.

This text is organized in the following way:

Section 2 is devoted to the background of the structures which will be used in the sequel.

In Section 3, starting from an almost contact metric structures we define metallic Riemannian structures and we investigate conditions for those structures being integrable and parallel then we give an example to confirm these latter properties.

In Section 4, we give the notion of metallic transformation and we use it for some questions of the characterization of certain geometric structures.

The Section 5 is devoted to give a generalization of the notion of metallic transformation which deduces the particular known cases.

In the last Section, we give an open question where we propose the first step to study the reverse, i.e. the construction of an almost contact metric structure starting from a metallic Riemannian structure.

\section{REVIEW OF NEEDED NOTIONS}

In this section, we give a brief information for metallic Riemannian manifolds and almost contact metric manifolds. We note that throughout this paper all manifolds and bundles, along with sections and connections, are assumed to be of class $C^{\infty}$.

Let $(M, g)$ be a Riemannian manifold. We present metallic Riemannian manifolds following [14]. A $(p, q)$-metallic structure on $M$ is a polynomial structure of second degree given by a $(1,1)$-tensor field $\Phi$ which satisfies

$$
\Phi^{2}=p \Phi+q I,
$$

where $I$ is the identity transformation and $p, q$ are fixed integers such that $x^{2}-$ $p x-q=0$ has a positive irrational root $\sigma_{p, q}$.

The number $\sigma_{p, q}$ is usually named a member of the metallic family. These numbers, denoted by:

$$
\sigma_{p, q}=\frac{p+\sqrt{p^{2}+4 q}}{2}
$$

are also called $(p, q)$-metallic numbers.

For example, we can talk about Golden structure if $p=1, q=1$ when the $\sigma_{1,1}$ is exactly the golden ratio $\phi=\frac{1+\sqrt{5}}{2}$, or about the silver structure $(p=2$, $\left.q=1, \sigma_{2,1}=1+\sqrt{2}\right)$, the bronze structure $\left(p=3, q=1, \sigma_{3,1}=\frac{3+\sqrt{13}}{2}\right)$, the nickel structure $\left(p=1, q=3, \sigma_{1,3}=\frac{1+\sqrt{13}}{2}\right)$, the copper structure $(p=1$, $q=2, \sigma_{1,2}=2$ ). The above numbers are closely related with different mathematical 
domains as dynamical systems, quasicristales, theory of Cantorial fractal-like microspace-time.

For the Riemannian manifold $(M, g)$ endowed with the $(p, q)$-metallic structure, we say that the metric $g$ is $\Phi$-compatible and that $M$ is a Riemannian metallic manifold [14, if

$$
g(\Phi X, Y)=g(X, \Phi Y)
$$

for all $X, Y$ vectors fiels on $M$. If we substitute $\Phi X$ into $X$ in (3), equation (3) may also written as

$$
g(\Phi X, \Phi Y)=g\left(\Phi^{2} X, Y\right)=g((p \Phi+q I) X, Y)=p g(\Phi X, Y)+q g(X, Y) .
$$

Here, we can show that such a metric always exists on a manifold with a metallic structure $\Phi$.

Proposition 1. If $(M, \Phi)$ is a metallic manifold, then $M$ admits a Riemannian metric $g$ such that

$$
g(\Phi X, Y)=g(X, \Phi Y) .
$$

Proof. Let $h$ be any Riemannian metric on $M$ and define $g$ by

$$
g(X, Y)=h(\Phi X, \Phi Y)+q h(X, Y),
$$

and check the details.

Note from Proposition 3.2 of [14] that every almost product structure $J$ induces two metallic structures on $M$ given as follows:

$$
\Phi_{1,2}=\frac{1}{2}\left(p I \pm\left(2 \sigma_{p, q}-p\right) J\right)
$$

is an almost product structure on $M$.

Conversely, every metallic structure $\Phi$ on $M$ induces two almost product structures on $M$ given as follows:

$$
J_{1,2}= \pm \frac{2 \Phi-p I}{2 \sigma_{p, q}-p}
$$

For a metallic manifold $(M, \Phi, g)$ and the associated almost product $J$, it is easy to see that

$$
g(J X, Y)=g(X, J Y),
$$

for every tangent vector fields $X$ on $M$.

In order that the Golden structure $\Phi$ is integrable, it is necessary and sufficient that it is possible to introduce a torsion-free affine connection $\nabla$ with respect to which the structure tensor $\Phi$ is covariantly constant. Also, we know that the integrability of $\Phi$ is equivalent to the vanishing of the Nijenhuis tensor $N_{\Phi}$ [14, where

$$
N_{\Phi}(X, Y)=\Phi^{2}[X, Y]+[\Phi X, \Phi Y]-\Phi[\Phi X, Y]-\Phi[X, \Phi Y]
$$

The link between the Nihenjuis tensors $\Phi$ and $J$ is given by

$$
N_{J}=\frac{4}{p^{2}+4 q} N_{\Phi}
$$


which show that the metallic structure $\Phi$ is integrable if and ony if the associated almost product $J$ is integrable.

An odd-dimensional Riemannian manifold $\left(M^{2 n+1}, g\right)$ is said to be an almost contact metric manifold if there exist on $M$ a $(1,1)$ tensor field $\varphi$, a vector field $\xi$ (called the structure vector field) and a 1-form $\eta$ such that

$$
\eta(\xi)=1, \varphi^{2}(X)=-X+\eta(X) \xi \quad \text { and } \quad g(\varphi X, \varphi Y)=g(X, Y)-\eta(X) \eta(Y),
$$

for any vectors fields $X, Y$ on $M$. In particular, in an almost contact metric manifold we also have

$$
\varphi \xi=0 \quad \text { and } \quad \eta \circ \varphi=0 .
$$

Such a manifold is said to be a contact metric manifold if

$$
d \eta=\Omega,
$$

where $\Omega(X, Y)=g(X, \varphi Y)$ is called the fundamental 2-form of $M$.

On the other hand, the almost contact metric structure of $M$ is said to be normal if

$$
N_{\varphi}(X, Y)=[\varphi, \varphi](X, Y)+2 d \eta(X, Y) \xi=0,
$$

for any $X$ and $Y$ vectors fields on $M$, where $[\varphi, \varphi]$ denotes the Nijenhuis torsion of $\varphi$, given by

$$
[\varphi, \varphi](X, Y)=\varphi^{2}[X, Y]+[\varphi X, \varphi Y]-\varphi[\varphi X, Y]-\varphi[X, \varphi Y] .
$$

An almost contact metric structure $(\varphi, \xi, \eta, g)$ on $M$ is said to be:

$$
\left\{\begin{array}{l}
(a): \text { Sasaki } \Leftrightarrow \Omega=\mathrm{d} \eta \text { and }(\varphi, \xi, \eta) \text { is normal, } \\
(b): \text { Cosymplectic } \Leftrightarrow \mathrm{d} \Omega=d \eta=0 \text { and }(\varphi, \xi, \eta) \text { is normal, } \\
(c): \text { Kenmotsu } \Leftrightarrow \mathrm{d} \eta=0, \mathrm{~d} \Omega=2 \Omega \wedge \eta \text { and }(\varphi, \xi, \eta) \text { is normal. }
\end{array}\right.
$$

where $\mathrm{d}$ denotes the exterior derivative.

In [20], the author proves that $(\varphi, \xi, \eta, g)$ is trans-Sasakian structure if and only if $(\varphi, \xi, \eta, g)$ is normal and

$$
d \eta=\alpha \Omega, \quad d \Omega=2 \beta \eta \wedge \Omega,
$$

where $\alpha=\frac{1}{2 n} \delta \Omega(\xi), \beta=\frac{1}{2 n} \operatorname{div} \xi$ and $\delta$ is the codifferential of g.

A trans-Sasakian structure $(\varphi, \xi, \eta, g)$ on $M$ is said to be

$$
\left\{\begin{array}{l}
(a): \alpha-\text { Sasaki if } \beta=0, \\
(b): \beta-\text { Kenmotsu if } \alpha=0, \\
(c): \text { Cosymplectic if } \alpha=\beta=0 .
\end{array}\right.
$$

(see [6], [18] and [23]).

The relation between trans-Sasakian, $\alpha$-Sasakian and, $\beta$-Kenmotsu structures was discussed by Marrero [19. 
Proposition 2. (Marrero [19])

A trans-Sasakian manifold of dimension $\geq 5$ is either $\alpha$-Sasakian, $\beta$-Kenmotsu or cosymplectic.

Proposition 3. (Marrero [19], Proposition 4.2)

Let $(M, \varphi, \xi, \eta, g)$ be a 3-dimensional Sasakian manifold. If we take $\bar{g}=f g+(1-$ $f) \eta \otimes \eta$ where $f>0$ a non-constant function on $M$ then,,$(M, \varphi, \xi, \eta, \bar{g})$ is a trans-Sasakian structure of type $\left(\frac{1}{f}, \frac{1}{2} \xi(\ln f)\right)$.

\section{Induced Metallic structures by almost contact structures}

In this section, starting from an almost contact metric structure we define a metallic Riemannian structure and we investigate conditions for those structures being integrable and parallel.

Theorem 4. Every almost contact metric structure $(\varphi, \xi, \eta, g)$ on a $(2 n+1)$ dimensional Riemannian manifold $(M, g)$ induces only two metallic structures on $(M, g)$, given as follows:

$$
\Phi_{1}=\sigma_{p, q} I+\left(p-2 \sigma_{p, q}\right) \eta \otimes \xi, \quad \Phi_{2}=\sigma_{p, q}^{*} I+\left(p-2 \sigma_{p, q}^{*}\right) \eta \otimes \xi,
$$

where $\xi$ is the unique eigenvector of $\Phi_{1}$ and $\Phi_{2}$ associated with $\sigma_{p, q}^{*}=p-\sigma_{p, q}$ and $\sigma_{p, q}$ respectively.

Proof. We try to write the metallic structure $\Phi_{i}$ with $i \in\{1,2\}$ defined on a $(2 n+1)$ dimensional Riemannian manifold $(M, g)$, using almost contact metric structure $(\varphi, \xi, \eta, g)$, in the form $\Phi=a_{i} I+b_{i} \eta \otimes \xi$, where $a_{i}$ and $b_{i}$ are non-zero constant. Thus

$$
\Phi^{2}=a_{i}^{2} I+b_{i}\left(2 a_{i}+b_{i}\right) \eta \otimes \xi
$$

and using formula (1) with $\Phi_{1} \xi=\sigma_{p, q}^{*} \xi$ and $\Phi_{2} \xi=\sigma_{p, q} \xi$, we obtain the formulas (16). Moreover, we have

$$
g\left(\Phi_{i} X, Y\right)=g\left(X, \Phi_{i} Y\right) \Leftrightarrow g(\varphi X, Y)=-g(X, \varphi Y),
$$

for every $i \in\{1,2\}$ and for every tangent vectors fields $X$ and $Y$ on $M$.

On the other hand, suppose that there exist another metallic structure on $M$ induces by the almost contact metric structure $(\varphi, \xi, \eta, g)$ denoted by $\Psi$ and admits $\xi$ as the unique eigenvector associated with $\sigma_{p, q}\left(\operatorname{resp} . \sigma_{p, q}^{*}\right)$ then, we have

$$
\Psi^{2}=p \Psi+q I, \quad \Psi \xi=\sigma_{p, q} \xi \quad\left(\text { resp. } \Psi \xi=\sigma_{p, q}^{*} \xi\right) .
$$

First, note that for all $i \in\{1,2\}$ we have

$$
\Phi_{i} \Psi=\Psi \Phi_{i}
$$

and using (1) and (17) we get

$$
\Psi^{2}-\Phi_{i}^{2}=p\left(\Psi-\Phi_{i}\right), \quad i=\overline{1,2}
$$


which gives

$$
\Psi=p-\Phi_{i} \in\left\{\Phi_{1}, \Phi_{2}\right\} .
$$

Remark 5. Using the two formulas in (16) we note that

$$
\Phi_{1}+\Phi_{2}=p I .
$$

Proposition 6. If $(M, \Phi, g)$ is a metallic Riemannian manifold, then $(M, \Phi, G)$ is also a metallic Riemannian manifold, where $G$ is a Riemannian metric given by:

$$
G(X, Y)=g(\varphi X, \varphi Y),
$$

for all vectors fields $X, Y$ on $M$.

Proof. Since the proof of the following proposition is obvious, we don't give the proof of it.

Using formula (4), we get the following:

Proposition 7. Every almost contact metric manifold $\left(M^{2 n+1}, \varphi, \xi, \eta, g\right)$ induces four almost product structures on $(M, g)$, given as follows:

$$
\begin{gathered}
J_{1}=I-2 \eta \otimes \xi, \quad J_{2}=I+2 \eta \otimes \xi, \\
J_{3}=-I+2 \eta \otimes \xi, \quad J_{4}=-I-2 \eta \otimes \xi .
\end{gathered}
$$

We note that through out this paper, we shall be setting

$$
\Phi=\sigma_{p, q} I+\left(p-2 \sigma_{p, q}\right) \eta \otimes \xi .
$$

Observe that,

$$
\sigma_{p, q}^{2}=p \sigma_{p, q}+q, \quad \sigma_{p, q}+\sigma_{p, q}^{*}=p \quad \text { and } \quad \sigma_{p, q} \cdot \sigma_{p, q}^{*}=-q .
$$

We know that the metallic structure $\Phi$ is integrable (i.e. $N_{\Phi}=0$ ) if and only if the almost product $J$ is integrable (i.e. $N_{J}=0$ ) with

$$
N_{J}(X, Y)=[X, Y]+[J X, J Y]-J[X, J Y]-J[J X, Y] .
$$

So, for all $X, Y$ vectors fields on $M$ and using (18), we get

$$
\frac{1}{8} N_{J}(X, Y)=(d \eta(X, Y)+\eta(X) d \eta(\xi, Y)+\eta(Y) d \eta(X, \xi)) \xi,
$$

witch give the following theorem:

Theorem 8. Let $\left(M^{2 n+1}, \Phi, g\right)$ be a metallic Riemannian manifold induced by the almost contact metric manifold $\left(M^{2 n+1}, \varphi, \xi, \eta, g\right)$. Then $\Phi$ is integrable if and only if $\eta$ is closed. 
Proof. Using the formula 20 with supposing that $d \eta=0$, we get $N_{J}=0$.

For the inverse, suppose that $N_{J}=0$. From 20) we have

$$
d \eta(X, Y)+\eta(X) d \eta(\xi, Y)+\eta(Y) d \eta(X, \xi)=0,
$$

taking $Y=\xi$ we obtain for all $X$ vector field on $M$,

$$
d \eta(X, \xi)=0
$$

Applying 22 in 211 we get

$$
d \eta(X, Y)=0,
$$

for all $X$ and $Y$ vectors fields tangent to $M$.

Remark 9. If $(M, \varphi, \xi, \eta, g)$ is an almost cosymplectic or an almost Kenmotsu manifold then $(M, \Phi, g)$ is an integrable metallic Riemannian manifold but for the contact case it is never integrable.

Lemma 10. If $(\Phi, g)$ is a metallic Riemannian structure induced by an almost contact metric structure $(\varphi, \xi, \eta, g)$ on $M$ then we have

$$
\varphi \Phi=\Phi \varphi=\sigma_{p, q} \varphi .
$$

Proof. Using formulas (19) and (10), the proof is direct.

Proposition 11. Let $\left(M^{2 n+1}, \Phi, g\right)$ be a metallic Riemannian manifold induced by the almost contact metric manifold $\left(M^{2 n+1}, \varphi, \xi, \eta, g\right)$. If $\nabla$ is the Levi-Cevita connection then for all $X$ and $Y$ vectors fields tangent to $M$ we have

$$
\left(\nabla_{X} \Phi\right) Y=\left(p-2 \sigma_{p, q}\right)\left(g\left(\nabla_{X} \xi, Y\right) \xi+\eta(Y) \nabla_{X} \xi\right) .
$$

Proof. From

$$
\left(\nabla_{X} \Phi\right) Y=\nabla_{X} \Phi Y-\Phi \nabla_{X} Y
$$

and using formula $(19)$, the proof is direct.

On the other hand, we know that the integrability of $\Phi$ is equivalent to the existence of a torsion-free affine connection with respect to which the equation $\nabla \Phi=0$ holds. Now we shall introduce another possible sufficient condition of the integrability of metallic structures on Riemannian manifolds.

Proposition 12. Let $\left(M^{2 n+1}, \Phi, g\right)$ be a metallic Riemannian manifold induced by the almost contact metric manifold $\left(M^{2 n+1}, \varphi, \xi, \eta, g\right)$. Then $\Phi$ is integrable (i.e. $\nabla \Phi=0$ ) if and only if $\nabla_{X} \xi=0$ for all $X$ vector field on $M$ where $\nabla$ is the the Levi-Cevita connection of $g$.

Proof. The necessity was observed above (see (24)). For the sufficiency, it suffices to replace $Y$ by $\xi$ in (24).

Remark 13. If $(\varphi, \xi, \eta, g)$ is a cosymplectic structure then $(\Phi, g)$ is a parallel metallic Riemannian structure. 
Example 14. For this example, we rely on our example in [1. We denote the Cartesian coordinates in a 3-dimensional Euclidean space $E^{3}$ by $(x, y, z)$ and define a symmetric tensor field $g$ by

$$
g=\left(\begin{array}{ccc}
\rho^{2}+\tau^{2} & 0 & -\tau \\
0 & \rho^{2} & 0 \\
-\tau & 0 & 1
\end{array}\right)
$$

where $\rho$ and $\tau$ are functions on $E^{3}$ such that $\rho \neq 0$ everywhere.

Further, we define an almost contact metric $(\varphi, \xi, \eta)$ on $E^{3}$ by

$$
\varphi=\left(\begin{array}{ccc}
0 & -1 & 0 \\
1 & 0 & 0 \\
0 & -\tau & 0
\end{array}\right), \quad \xi=\left(\begin{array}{l}
0 \\
0 \\
1
\end{array}\right), \quad \eta=(-\tau, 0,1) .
$$

Using the formula 19) we get

$$
\Phi=\left(\begin{array}{ccc}
\sigma_{p, q} & 0 & 0 \\
0 & \sigma_{p, q} & 0 \\
\tau\left(2 \sigma_{p, q}-p\right) & 0 & 1-\sigma_{p, q}
\end{array}\right)
$$

where we can check that $\Phi^{2}=p \Phi+q I$.

The fundamental 1-form $\eta$ have the form,

$$
\eta=d z-\tau d x,
$$

and hence

$$
d \eta=\tau_{2} d x \wedge d y+\tau_{3} d x \wedge d z .
$$

With a straightforward computation, one can get

$\nabla_{\partial x} \xi=\frac{1}{\rho^{2}}\left(\begin{array}{c}\rho \rho_{3}+\tau \tau_{3} \\ \tau_{2} \\ \tau \rho \rho_{3}+\tau \tau_{3}\end{array}\right) ; \quad \nabla_{\partial y} \xi=\frac{1}{\rho^{2}}\left(\begin{array}{c}-\tau_{2} \\ \rho_{3} \\ -\tau \tau_{2}\end{array}\right) ; \quad \nabla_{\partial z} \xi=\frac{1}{\rho^{2}}\left(\begin{array}{c}-\tau_{3} \\ 0 \\ -\tau \tau_{3}\end{array}\right)$,

where $\rho_{i}=\frac{\partial \rho}{\partial x_{i}}$ and $\tau_{i}=\frac{\partial \tau}{\partial x_{i}}$.

On the other hand, according to the cases given in [1, the structure $(\varphi, \xi, \eta, g)$ is $a$ :

(1) Cosymplectic when $\rho_{3}=\tau_{2}=\tau_{3}=0$,

(2) Kenmotsu when $\rho_{3}=\rho, \tau_{2}=0$ and $\tau_{3}=0$.

So,

(a) If $\tau_{2}=\tau_{3}=0$ (i.e. $d \eta=0$ ) then the metallic structure $\Phi$ is integrable.

(b) If $\rho_{3}=\tau_{2}=\tau_{3}=0$ (i. e. $\left.\nabla_{X} \xi=0\right)$ then the metallic structure $\Phi$ is parallel. 


\section{Metallic transformation}

Let $\left(M^{2 n+1}, \Phi, g\right)$ be a metallic Riemannian manifold induced by the almost contact metric manifold $\left(M^{2 n+1}, \varphi, \xi, \eta, g\right)$.

We mean a change of structures tensors of the form

$$
\begin{gathered}
\tilde{\varphi}=\varphi, \quad \tilde{\xi}=\frac{1}{p-\sigma_{p, q}} \xi, \quad \tilde{\eta}=\left(p-\sigma_{p, q}\right) \eta, \\
\tilde{g}(X, Y)=g(\Phi X, \Phi Y)=\sigma_{p, q}^{2} g+p\left(p-2 \sigma_{p, q}\right) \eta \otimes \eta .
\end{gathered}
$$

Proposition 15. If $(\varphi, \xi, \eta, g)$ is an almost contact metric structure, then $(\tilde{\varphi}, \tilde{\xi}, \tilde{\eta}, \tilde{g})$ is also an almost contact metric structure.

Proof. Obvious (using formulas (9)).

We refer to this construction as metallic deformation.

Theorem 16. Let $(M, \varphi, \xi, \eta, g)$ be an almost contact metric manifold and $(M, \tilde{\varphi}, \tilde{\xi}, \tilde{\eta}, \tilde{g})$ is an almost contact metric manifold obtained as above, then it is:

(a) $\alpha$-Sasaki with $\alpha=\frac{p-\sigma_{p, q}}{\sigma_{p, q}^{2}}$ if and only if $(M, \varphi, \xi, \eta, g)$ is a Sasakian manifold.

(b) $\beta$-Kenmotsu with $\beta=\frac{1}{p-\sigma_{p, q}}$ if and only if $(M, \varphi, \xi, \eta, g)$ is a Kenmotsu manifold.

(c) Cosymplectic if and only if $(M, \varphi, \xi, \eta, g)$ is a cosymplectic manifold.

Proof. Let $(M, \varphi, \xi, \eta, g)$ be a trans-Sasakian manifold of type $(\alpha, \beta)$. The fundamental 1-form $\tilde{\eta}$ and the 2-forme $\tilde{\Omega}$ of the structure $(\tilde{\varphi}, \tilde{\xi}, \tilde{\eta}, \tilde{g})$ defined as above have the forms,

$$
\tilde{\eta}=\left(p-\sigma_{p, q}\right) \eta \quad \text { and } \quad \tilde{\Omega}=\sigma_{p, q}^{2} \Omega
$$

where $\Omega$ is the 2 -form of the almost contact metric structure $(\varphi, \xi, \eta, g)$ and hence

$$
\mathrm{d} \tilde{\eta}=\left(p-\sigma_{p, q}\right) \mathrm{d} \eta \quad \text { and } \quad \mathrm{d} \tilde{\Omega}=\sigma_{p, q}^{2} \mathrm{~d} \Omega,
$$

using the formulas 14 we get

$$
\mathrm{d} \tilde{\eta}=\frac{p-\sigma_{p, q}}{\sigma_{p, q}^{2}} \alpha \tilde{\Omega} \quad \text { and } \quad \mathrm{d} \tilde{\Omega}=\frac{2 \beta}{p-\sigma_{p, q}} \tilde{\eta} \wedge \tilde{\Omega} .
$$

Knowing that the trans-Sasakian manifolds of type $(1,0),(0,1)$ and $(0,0)$ are called Sasakian, Kenmotsu and cosymplectic manifolds respectively then the proof is completed.

Remark 17. Note that the metallic transformation preserve the structure cosymplectic for all two positive integers $p$ and $q$ and the Kenmotsu structure only for $q=p+1$ but the Sasakian structure is never preserved.

A straightforward computation yields the following proposition: 
Proposition 18. If $(\Phi, g)$ be a metallic Riemannian structure induced by the almost contact metric structure $(\varphi, \xi, \eta, g)$, then the structure $(\widehat{\varphi}, \widehat{\xi}, \widehat{\eta}, \widehat{g})$ given by

$$
\begin{gathered}
\widehat{\varphi}=\varphi, \quad \widehat{\xi}=\frac{1}{\left(p-\sigma_{p, q}\right)^{n}} \xi, \quad \widehat{\eta}=\left(p-\sigma_{p, q}\right)^{n} \eta, \\
\widehat{g}(X, Y)=g\left(\Phi^{n} X, \Phi^{n} Y\right)=\sigma_{p, q}^{2 n} g+\left(\sigma_{p, q}^{2 n}-\left(p-\sigma_{p, q}\right)^{2 n}\right) \eta \otimes \eta .
\end{gathered}
$$

for any integer number $n$, is also an almost contact metric structure.

\section{Generalized $\mathcal{D}$-homothetic transformation}

Let $(M, \varphi, \xi, \eta, g)$ be an almost contact metric manifold with $\operatorname{dim} M=2 n+1$. The equation $\eta=0$ defines a $2 n$-dimensional distribution $\mathcal{D}$ on $M$. By an $2 n$ homothetic deformation or $\mathcal{D}$-homothetic deformation [22] we mean a change of structure tensors of the form

$$
\bar{\varphi}=\varphi, \quad \bar{\eta}=a \eta, \quad \bar{\xi}=\frac{1}{a} \xi, \quad \bar{g}=a g+a(a-1) \eta \otimes \eta,
$$

where $a$ is a positive constant. If $(M, \varphi, \xi, \eta, g)$ is a contact metric structure with contact form $\eta$, then $(\bar{M}, \bar{\varphi}, \bar{\xi}, \bar{\eta}, \bar{g})$ is also a contact metric structure [22].

This idea works equally well for almost contact metric structures. The deformation

$$
\tilde{\varphi}=\varphi, \quad \tilde{\xi}=\frac{1}{h} \xi, \quad \tilde{\eta}=h \eta, \quad \tilde{g}(X, Y)=f^{2} g+\left(h^{2}-f^{2}\right) \eta \otimes \eta,
$$

is again an almost contact metric structure where $f$ and $h$ are two non-zero functions on $M$.

From the theorem (16), we can deduce the following proposition:

Proposition 19. Let $(M, \varphi, \xi, \eta, g)$ be an almost contact metric manifold and $(M, \tilde{\varphi}, \tilde{\xi}, \tilde{\eta}, \tilde{g})$ is an almost contact metric manifold obtained as above, then it is:

(a) $\alpha$-Sasaki with $\alpha=\frac{h}{f^{2}}$ and $h$ is constant if and only if $(M, \varphi, \xi, \eta, g)$ is a Sasakian manifold.

(b) $\beta$-Kenmotsu with $\beta=\frac{1}{h}$ with $f$ is constant if and only if $(M, \varphi, \xi, \eta, g)$ is a Kenmotsu manifold.

(c) Cosymplectic where $f, h$ are constant if and only if $(M, \varphi, \xi, \eta, g)$ is a cosymplectic manifold.

\section{Special cases:}

- For $h= \pm f$, we get the conformal transformation [21].

- For $h=f^{2}$ and $f=$ constant, we get the deformation of Tanno [22].

- For $h= \pm 1$, we get the deformation of Marrero [19].

- For $f= \pm 1$, we get the $\mathcal{D}$-isometric [2]. 


\section{OPEN PROBLEM}

Finally, we propose the first steps to construct an almost contact metric structure from a metallic Riemannian structure.

Let $\left(M^{2 n+1}, \Phi, g\right)$ be a metallic Riemannian manifold and $\xi$ be the unique eigenvector of $\Phi$ associated with $\sigma_{p, q}^{*}=p-\sigma_{p, q}\left(\operatorname{resp} . \sigma_{p, q}\right)$ which give $\Phi \xi=\sigma_{p, q}^{*} \xi($ resp. $\left.\Phi \xi=\sigma_{p, q} \xi\right)$ and let $\eta$ be the $g$-dual of $\xi$ i.e. $\eta(X)=g(X, \xi)$ for all vector field $X$ on $M$ such that $\eta(\xi)=1$.

Proposition 20. The metallic structure $\Phi$ admits the following expression:

$$
\Phi=\sigma_{p, q} I+\left(p-2 \sigma_{p, q}\right) \eta \otimes \xi, \quad\left(\text { resp. } \quad \Phi=\sigma_{p, q}^{*} I+\left(p-2 \sigma_{p, q}^{*}\right) \eta \otimes \xi\right),
$$

Proof. We try to write the metallic structure $\Phi$ in the form $\Phi=a I+b \eta \otimes \xi$, where $a, b \in \mathbb{R}^{*}$. Thus

$$
\Phi^{2}=a^{2} I+b\left(a+\sigma_{p, q}^{*}\right) \eta \otimes \xi
$$

on the other hand, we have

$$
p \Phi+q I=(a p+q) I+p b \eta \otimes \xi,
$$

using formulas (1) we obtain the formulas (27).

One can construct on $M^{2 n+1}$ an almost contact metric structure $(\varphi, \xi, \eta, g)$ starting from a metallic Riemannian structure and study its nature taking into account the two parameters $p$ and $q$.

Acknowledgement. The author would like to thank the referees for their helpful suggestions and their valuable comments which helped to improve the manuscript.

\section{REFERENCES}

[1] Beldjilali, G., Belkhelfa, M. , Kählerian structures on D-homothetic bi-warping, J. Geom. Symmetry Phys. 42 (2016), 1-13.

[2] Beldjilali, G., Structures and $\mathcal{D}$-isometric warping, HSJG, 2(1) (2020), 21-29 .

[3] Beldjilali, G., Induced structures on Golden Riemannian manifolds., Beitr Algebra Geom., 59 (2018), 761-777.

[4] Beldjilali, G., s-Golden Manifolds, Mediterr. J. Math., (2019), https://doi.org/10.1007/s00009-019-1343-9.

[5] Beldjilali, G., A new class of Golden Riemannian manifold. Int. Electron. J. Geom., 13(1) (2020), 1-8.

[6] Blair, D. E. , Riemannian Geometry of Contact and Symplectic Manifolds, Progress in Mathematics, Vol. 203, Birhauser, Boston, 2002

[7] Acet, B. E., Lightlike hypersurfaces of metallic semi-Riemannian manifolds, Int. J. of Geo. Meth. in Mod. Phys., Vol. 15 (2018), 185-201 .

[8] Crasmareanu, M., Hretecanu, C.E., Golden differential geometry, Chaos, Solitons 83 Fractals, 38 (2008), 1124-1146. doi: 10.1016/j.chaos.2008.04.007.

[9] Debmath, P, Konar, A., A new type of structure on differentiable manifold, Int. Electron. J. Geom., 4 (2011), 102-114.

[10] De Spinadel, V.W., The metallic means family and the multifractal, spectra. Nonlinear Anal., 36(6) (1999), 721-745. 
[11] Etayo, F., Santamaria, R. and Upadhyay, A., On the Geometry of Almost Golden Riemannian Manifolds, Mediterr. J. Math.,14 187 (2017). https://doi.org/10.1007/s00009-017-0991-x.

[12] Hrețcanu, C.E.: Submanifolds in Riemannian manifold with Golden structure. Workshop on Finsler Geometry and its Applications, Hungary, (2007).

[13] Hrețcanu C.E., and Blaga A.M., Hemi-slant submanifolds in metallic Riemannian manifolds, Carpathian J., 35 (1) (2019), 59-68.

[14] Hrețcanu C.E., Crasmareanu M., Metallic structures on Riemannian Manifolds, Rev Un Mat Argentina. 54 (2013), 15-27.

[15] Gezer A., Cengiz N., Salimov A., On integrability of Golden Riemannian structures, Turkish J. Math., 37 (2013), 693-703.

[16] Gezer A., Karaman C., Golden-Hessian structures, Proceedings of the National Academy of Sciences, India Section A: Physical Sciences, 86 (1) (2016), 41-46, .

[17] Gönül S., Küpeli Erken I., Yazla A. and Murathan C., A Neutral relation between metallic structure and almost quadratic $\varphi$-structure, Turkish Journal of Mathematics, 43 (2019), 268278.

[18] Kenmotsu K., A class of almost contact Riemannian manifolds, J. Tohoku Math., 24 (1972), 93-103.

[19] Marrero J. C., The local structure of trans-Sasakian manifolds, Annali di Matematica Pura ed Applicata 162(1) (1992), 77-86.

[20] Oubiña, J.A., New classes of almost contact metric structures, Publicationes Mathematicae, Debrecen, 32 (1985), 187-193.

[21] Sharfuddin, A., Husain, S. I., Almost contact structures induced by conformal transformation, Publ. Inst. Math. Nouv., 32 (46) (1982), 155-159.

[22] Tanno S., The topology of contact Riemannian manifolds, Illinois J. Math., 12 (1968), 700717.

[23] Yano, K., Kon, M., Structures on Manifolds, Series in Pure Math., Vol 3, World Sci.,1984. 